\title{
ELEMENTS AND EPHEMERIS OF JUPITER'S SIXTH SATELLITE
}

The observations of Jupiter's Sixth Satellite which have been secured up to the present time, extend over too short an interval to enable the elements of its orbit to be obtained with any great accuracy, notwithstanding the undoubted precision of the observations. With the exception of its period of revolution, which will be somewhat uncertain, it is believed, however, that a very good first approximation to these elements can be now obtained. They should prove ample for the construction of a finding ephemeris for the next opposition.

The orbit which has been obtained for this satellite, and which appears below, is based upon eight observations secured by Professor Perrine at the Lick Observatory. They cover the entire period of visibility, from discovery until its conjunction with the Sun in the early spring. The dates of these observations are, respectively, December 3, December 10, 1904; January 3, January 28, February 8, February 22, February 28, and March 6, 1905 . It will not be necessary to go into the details of the investigation of its orbit. The elements which were obtained are as follows :

Elements Referred to Terrestrial Equator.

\begin{tabular}{|c|c|c|}
\hline Mean Jovicentric Right Ascension & $288^{\circ} 76$ & \\
\hline Right Ascension of Perijove & $257^{\circ} .0$ & \\
\hline Right Ascension of Node on Equator & $176^{\circ} .7$ & \\
\hline Inclination to Equator & $5^{\circ} 29^{\prime}$ & \\
\hline Echiptic Elements. & & 1905 \\
\hline Mean Longitude & $288^{\circ} .71$ & \\
\hline Longitude of Perijove & $256^{\circ} .95$ & \\
\hline Longitude of Node on Ecliptic & $179^{\circ} .35$ & \\
\hline Inclination to Ecliptic & $28^{\circ} 56^{\prime}$ & \\
\hline Inclination to Jupiter's Orbit & $28^{\circ} 44^{\prime}$ & \\
\hline Mean Daily Tropical Motion & \\
\hline Mean Distance $(\log \Delta=0.71624)$ & \multicolumn{2}{|c|}{$2970^{\prime \prime}$} \\
\hline Eccentricity & \multicolumn{2}{|c|}{0.1564} \\
\hline Period & \multicolumn{2}{|c|}{242.4 days } \\
\hline
\end{tabular}

The residuals for the eight observations which these elements give are, respectively,

In Right Ascension:

$+0.4,-6.4,-2.8,+4.3,+7.2,+1.6,-2.6,-6.2$

In Declination:

$+1 " 3,-0.6,+0.4,-1 " 2,+0.9,-0.5,+1{ }^{\prime \prime} 1,+0.1$

It would seem at first sight, from an inspection of the residuals in right ascension, that an improvement on these elements could be obtained. It was found, however, that the arc covered by the observations was too small to enable the method of differential corrections to be successfully applied. Again, and what is of more importance, the large perturbations to which the satellite is subject can easily account for the residuals still outstanding. (See below).

The smallness of the residuals in declination show conclusively that the motion is in a direct orbit, in accordance with the above elements.

\section{SOLAR PERTURBATIONS.}

The perturbations suffered by this satellite, due to the Sun's action, are larger and more complex than that of any other known satellite in the solar system. On account of its great distance from its primary, amounting, at the maximum, to $76^{\prime}$, it is found that perturbations of the jovicentric longitude of the satellite of as small an amount as 4 " will be perceptible to observation. In order to construct an accurate theory of its motion, at least 50 inequalities in the longitude, as many more in the radius vector, and about 20 in the latitude, will have to be computed. It can therefore be taken out of the class of ordinary satellites, and, in this respect, will rank with the major planets and asteroids, requiring the use of extensive tables in all computations relative to its motion. There is, however, one other satellite, namely, Phœbe, the ninth satellite of Saturn, recently discovered by Professor W. H. Pickering, which has a similar complex theory. These two therefore form a class by themselves.

It may be of interest to state the values of some of the principal solar perturbations. By applying Delaunay's general theory, I get the following results :

$\begin{array}{lr}\text { Annual Motion of Node on Jupiter's Orbit } & -1: 16 \\ \text { Annual Motion of Node on Earth's Equator } & -4: 75 \\ \text { Annual Motion of Perijove } & +1: 25 \\ & \\ \text { Coefficient of Annual Equation } & +0.34 \\ \text { Coefficient of Evection } & +2: 14 \\ \text { Coefficient of Variation } & +0.61\end{array}$

There are corespondingly large perturbations of the radius vector; also, three inequalities in latitude with coefficients larger than $0: 6$. In order to give some idea of the effect of these perturbations upon the apparent position of the satellite, it may be stated that the evectional inequality alone will produce a 
maximum geocentric displacement of the satellite of over three minutes of are.

The perturbing action of Saturn will be small, not amounting at the maximum to more than $1 " 0$.

On account of the great distance of the satellite from Jupiter, it should prove useful in obtaining a new determination of the mass of that body. The differences existing between the best determinations of Jupiter's mass so far made, correspond to an error of 0 "65 in the measurement of the sixth satellite's major axis, so that very good results should be obtained from such a determination.
EPHIEMERIS OF SATELLITE VI.

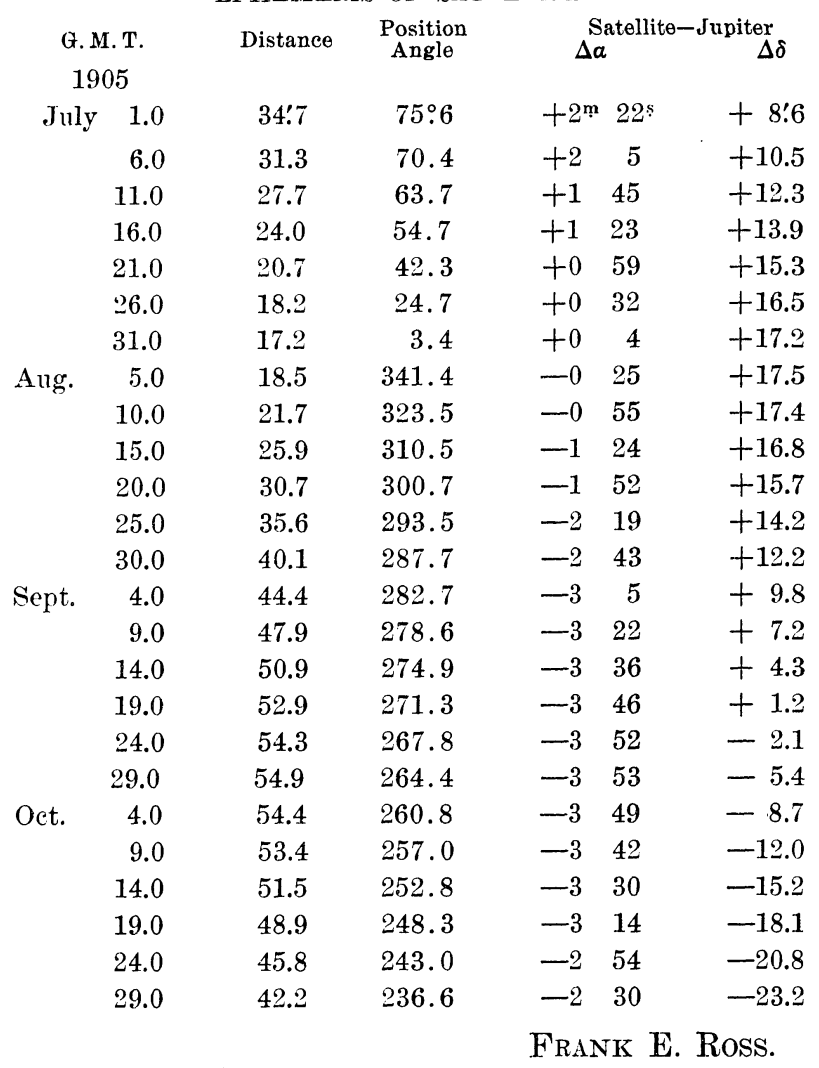

May 10, 1905. 